\title{
Graded Carrier Concentration Absorber Profile for High Efficiency CIGS Solar Cells
}

\author{
Antonino Parisi, Riccardo Pernice, Vincenzo Rocca, Luciano Curcio, \\ Salvatore Stivala, Alfonso C. Cino, Giovanni Cipriani, Vincenzo Di Dio, \\ Giuseppe Ricco Galluzzo, Rosario Miceli, and Alessandro C. Busacca
}

DEIM, University of Palermo, Viale delle Scienze, Building 9, 90128 Palermo, Italy

Correspondence should be addressed to Alessandro C. Busacca; alessandro.busacca@unipa.it

Received 28 November 2014; Revised 9 April 2015; Accepted 19 April 2015

Academic Editor: Cheuk-Lam Ho

Copyright (C) 2015 Antonino Parisi et al. This is an open access article distributed under the Creative Commons Attribution License, which permits unrestricted use, distribution, and reproduction in any medium, provided the original work is properly cited.

We demonstrate an innovative CIGS-based solar cells model with a graded doping concentration absorber profile, capable of achieving high efficiency values. In detail, we start with an in-depth discussion concerning the parametrical study of conventional CIGS solar cells structures. We have used the wxAMPS software in order to numerically simulate cell electrical behaviour. By means of simulations, we have studied the variation of relevant physical and chemical parameters-characteristic of such devices-with changing energy gap and doping density of the absorber layer. Our results show that, in uniform CIGS cell, the efficiency, the open circuit voltage, and short circuit current heavily depend on CIGS band gap. Our numerical analysis highlights that the band gap value of $1.40 \mathrm{eV}$ is optimal, but both the presence of Molybdenum back contact and the high carrier recombination near the junction noticeably reduce the crucial electrical parameters. For the above-mentioned reasons, we have demonstrated that the efficiency obtained by conventional CIGS cells is lower if compared to the values reached by our proposed graded carrier concentration profile structures (up to $21 \%$ ).

\section{Introduction}

Quaternary chalcopyrite semiconductor alloy $\mathrm{Cu}(\mathrm{In}, \mathrm{Ga}) \mathrm{Se}_{2}$ (CIGS) represents one of the most suitable materials to produce low-cost and high-efficiency photovoltaic modules and hence can be considered as an appropriate alternative to the silicon technology. Recently, CIGS solar cells have gathered a noteworthy attention, since they possess a direct band gap falling within the range useful for several important applications, such as solid state lighting and high-efficiency photovoltaic modules [1]. Specifically, thin-film CIGS solar cells have emerged as a technology that could challenge the current dominance of silicon solar cells. This is possible thanks to the peculiar optical and structural properties of CIGS cells, which possess an extraordinary stability under a wide range of operating conditions. Nowadays, CIGS technology is industrially well exploited and commercial modules are broadly available.

In detail, this semiconductor presents a band gap tunable through the progressive Gallium incorporation, varying from
1.02 to $1.68 \mathrm{eV}$. Potentially, a wide band gap of the absorber designed to match the solar spectrum represents an effective way to increase the efficiency. Moreover, in this manner the open-circuit voltage $\left(V_{\mathrm{OC}}\right)$ can be increased, thus allowing to minimize the number of cells in series in the final assembled panel, and hence the interconnection losses.

In the literature, several research groups have demonstrated the realization of CIGS cells having conversion efficiencies of over $20 \%$, quite similar to the value obtained by crystalline silicon cells, that is, around 25\% [2]. A great deal of research is devoted to improve the performance, and this can only be achieved starting from a better understanding of the basic properties and of the common challenges that can limit the electrical parameters. For example, several papers in the literature reported on CIGS solar cells presenting lateral inhomogeneities on the $\mu \mathrm{m}$ scale that affect the main parameters, in particular the local splitting of quasi-Fermi levels obtained from photoluminescence measurements and thus decrease the overall performance [3]. Reducing or avoiding these problems is of great importance, since it is 
worth noting that the direct conversion of sunlight into electricity, that is, photovoltaics, can be surely considered as a fundamental alternative to fossil fuels and could become, as experts say, "the biggest supplier of electricity by the end of the century" [4]. For this reason, over the last decades, many efforts have been made in this research field, in particular to accurately control the composition of deposited absorbers material. In addition, it is worth noting that the technological requirements to obtain reproducible and controllable compositions are in common with optical applications previously developed in different research environments [5-9].

Finally, it is worth mentioning that, from a broader perspective, interesting results can be expected from even more complex materials, for example, those based on pentanary $\mathrm{Cu}(\mathrm{In}, \mathrm{Ga})(\mathrm{S}, \mathrm{Se})_{2}$ alloys [10] or comprising nanostructured layers/contacts [11]. For example, in [11], the authors have developed $\mathrm{Al}_{2} \mathrm{O}_{3}$ rear surface passivated CIGS solar cells with the local rear contacts consisting of Mo nanoparticles, in order to improve the optical confinement and hence to obtain an increased short circuit current density, if compared to CIGS solar cells with a standard back contact.

In literature, it has been reported that nonuniform $\mathrm{Ga} / \mathrm{In}$ composition versus the absorber depth can improve the device performance [12]. The Ga fraction [i.e., $x=$ $([\mathrm{Ga}] /[\mathrm{Ga}]+[\mathrm{In}])]$ influences the CIGS electron affinity, changing the value of $E_{c}$, while $E_{v}$ is almost independent of $x$. [13]. The three most used typologies of grading are (i) linear front grading, in which the Ga fraction content increases in $z$ (i.e., the depth) towards the junction; (ii) back grading, in which the increase is towards the back contact; (iii) double grading, in which the Ga first decreases from the front surface to a minimum position inside the absorber (i.e., in the centre of the structure) and then increases again towards the back contact [12]. The back grading causes the band gap of the absorber to linearly increase towards the back contact. Therefore, back grading creates an extra drift field for the minority electrons that improves the carrier collection and decreases bulk and surface recombination in the back contact interface. The $V_{\mathrm{OC}}$ of the cell is also augmented due to a lower recombination current (saturation current) [14]. However, a disadvantage consists in the Short-Circuit Current Density $\left(J_{\mathrm{SC}}\right)$ which does not efficiently exploit the solar spectrum. Instead, in a linear front grading, the gradual reduction of the Ga content through the absorber layer causes the band gap to linearly decrease with the depth. In this way, $J_{\mathrm{SC}}$ increases due to the augmented photon absorption for lower band gaps (i.e., thermalization losses are reduced). However, this increase can be negligible due to a concurrent reduced probability of the electron collection caused by the reverse quasielectrical field [14]. A double graded profile allows increased performance since the front grading improves the open-circuit voltage of the cell, while the back grading favours the collection of photo generated carriers, enhancing the photocurrent [12]. The maximum band gap at the back contact should be optimized to achieve the desired improved $J_{\mathrm{SC}}[14]$.

In this paper, we show a simulative analysis aimed at investigating the possible effects of both tailoring the absorber band gap and changing the carrier concentration profile on the main electrical parameters of CIGS based solar cells, in order to design graded absorber profiles that are able to increase the efficiency. This has been performed to simulate structures suitable to be realized within an ongoing experimental activity. The technology of choice is single-step electrodeposition [15-18], which allows to finely adjust Ga content up to the found optimum band gap value of the CIGS absorber. During our simulations, performed using the wxAMPS software, we have first analysed five band gap values at varying absorber thickness, and then we have focused on a novel graded linear absorber profile, as will be illustrated in the following sections.

\section{Numerical Modelling and Material Parameters}

2.1. Numerical Analysis of CIGS Cells. CIGS polycrystalline solar cells are quite complex structures. This is due to the fact that they are composed of a large number of layers and, moreover, the effects of some phenomena, mechanisms or material parameters often cannot be directly derived from theoretical laws. Conversely, an empirical approach has to be followed. In order to understand and explain the results obtained through experimental measurements and, furthermore, try to solve the problems, numerical simulations have to be exploited. The latter are also carried out to clarify the potential advantages of a certain cell structure or the limits imposed by a specific technology. In particular, by means of simulations, it is possible to quantitatively describe the measurements carried out, thus providing an in-depth analysis of the physical behaviour of the cell. Moreover, it is possible to evaluate how the variation of some parameters of the used material influences the solar cell [19]. In fact, the variation of the considered properties in the ranges of acceptable values can strongly change the efficiency of the solar cell under study. By performing a parametric study, it is thus possible to determine the best values that optimize the efficiency, giving the experimental researcher some important information about fabrication methods to use, in order to improve device and product performances.

Several software solutions, such as Analysis of Microelectronic and Photonic Structures (AMPS-1D) [20], wxAMPS (Analysis of Microelectronic and Photonic Structure, wXwidgets provided) [21], Photovoltaic cell 1-Dimension (PC1D) [22], and Solar Cell Capacitance Simulator in 1 Dimension (SCAPS-1D) [23], have been developed to perform an indepth simulation study of the functioning of multilayer structures, including those used for our thin film solar cells.

In this study, we have conducted our investigations extensively using wxAMPS, a freeware solar-cell simulation software. This program is an enhanced version, rewritten in $\mathrm{C}++$ by Rockett et al. at the University of Illinois, of the previous AMPS-1D software, as developed by Fonash et al. at Pennsylvania State University [21, 24]. wxAMPS allows the simulation of optical and electronic behavior for multiple designs of solar cell devices. By means of this software, it is possible to easily design the solar cell structure and to properly select the input parameters. Subsequently, the computation of many parameters and characteristics, such 
as J-V curves, fill-factor, efficiency, energy band diagrams, electric field, density carrier, generation, and recombination velocity, can be easily and automatically carried out.

wxAMPS versatility has also allowed us to investigate, in detail, the optical responses and the electrical transport phenomena of envisioned structures in our solar cells. A very large number of layers, with any kind of combination and composition can be engineered, to simulate the cross section of any feasible device. Sophisticated algorithms, such as the combination of the Gummel and Newton methods, are used in wxAMPS for the simulations. The advantages consist in an increased stability and a more consistent convergence in those instances in which intraband tunneling is critical to calculate specific solutions [25]. Finally, wxAMPS allows to setting, by means of a complete and user-friendly graphical interface, the environmental conditions and the physical properties of each layer. A good starting set of simulation parameters is provided by the University of Illinois, Engineering Wiki website [26], but it is also possible to freely customize the database by means of worksheets.

2.2. Stacked Structure. In this paper, we refer to a solar cell of the type $\mathrm{ZnO}: \mathrm{Al} / \mathrm{ZnO} / \mathrm{CdS} / \mathrm{CIGS} / \mathrm{Mo} /$ substrate, as sketched in Figure 1(a).

The most important layer of the abovementioned photovoltaic cell is the CIGS absorber. In our work, we have first considered a single absorber layer with constant properties (i.e., doping concentrations, energy gap, etc.), as in conventional CIGS solar cells. Afterwards, we have taken into account a newly proposed absorber structure (Figure 1(b)), consisting of several different layers having each one a different doping concentration $\left(N_{A}\right)$. In detail, we have considered a linearly increasing doping concentration profile, with the maximum value of $N_{A}$ in the layer located near the absorberMolibdenum interface.

With regard to the CdS buffer layer, it is known that $\mathrm{CdS}$ is a direct band gap semiconductor (band-gap $=2.4 \mathrm{eV}$ ) and its use as a buffer layer improves the performance of the solar cell, since it provides a lower interface recombination, the prevention of undesirable shunt paths through the absorber and less structural damage due to the subsequent deposition of $\mathrm{ZnO}$ onto CIGS. Moreover, CdS layer also reduces reflection losses at the cell surface, since its refractive index falls between those of $\mathrm{ZnO}$ and CIGS.

As evident in Figure 1(a) a p-n junction is formed by the $\mathrm{ZnO}$-CdS (n-type material) with p-type CIGS. The thickness of CdS is usually chosen within the $50-100 \mathrm{~nm}$ range, since this value represents the best compromise between the abovementioned advantages and losses due to optical absorption. On the top of the buffer layer, an $\mathrm{n}$-doped $\mathrm{ZnO}$ and an aluminium-doped $\mathrm{ZnO}$ ( $\mathrm{ZnO}: \mathrm{Al})$ layers are deposited.

These two layers form the so-called transparent conductive oxide (TCO), since they both possess a wider band-gap, so being transparent to most of the solar spectrum. The most important layer in the photovoltaic cell is the absorber one, represented by the CIGS layer. $\mathrm{Cu}(\mathrm{In}, \mathrm{Ga}) \mathrm{Se}_{2}$ is a semiconductor compound exhibiting a chalcopyrite crystal structure and possessing a high absorption coefficient $\left(\alpha \sim 10^{5} \mathrm{~cm}^{-1}\right)$ [27].

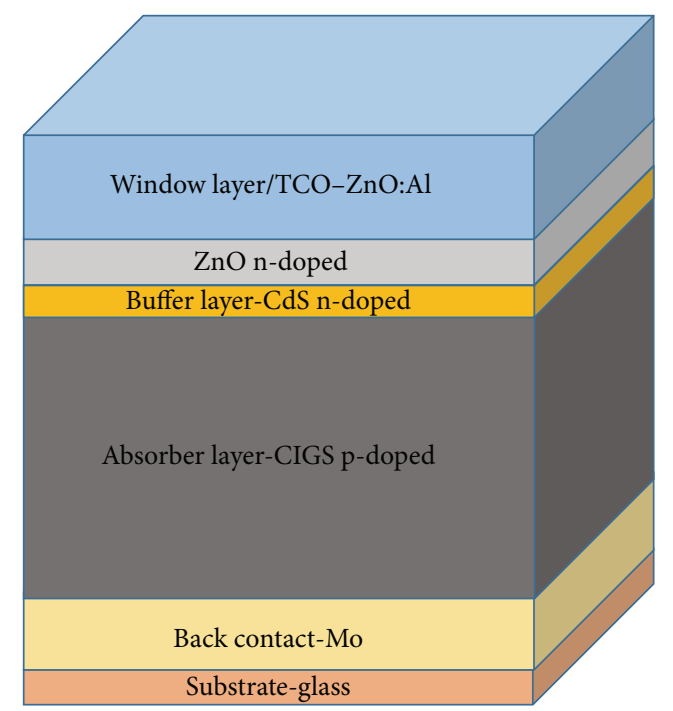

(a)

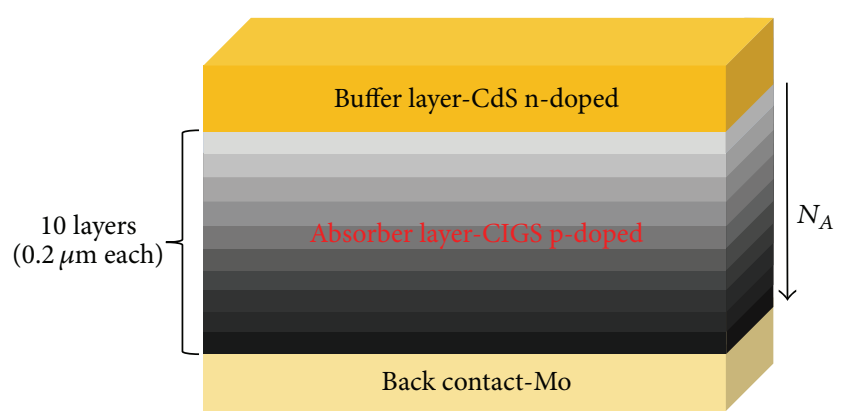

(b)

FIGURE 1: (a) Structure of CIGS-based solar cells (drawing not to scale); (b) graded absorber profile structure.

TABLE 1: Simulation material parameters of the CIGS thin-film solar cell.

\begin{tabular}{lcccc}
\hline Parameter & $\mathrm{ZnO}: \mathrm{Al}$ & $\mathrm{ZnO}$ & $\mathrm{CdS}$ & CIGS \\
\hline$d(\mu \mathrm{m})$ & 0.2 & 0.2 & 0.05 & Variable \\
$\varepsilon_{R}$ & 9 & 9 & 10 & 13.6 \\
$E_{g}(\mathrm{eV})$ & 3.3 & 3.3 & 2.4 & Variable \\
$\chi(\mathrm{eV})$ & 4.4 & 4.4 & 4.2 & Variable \\
$N_{C}\left[\mathrm{~cm}^{-3}\right]$ & $2.2 \times 10^{18}$ & $2.2 \times 10^{18}$ & $2.2 \times 10^{18}$ & $2.2 \times 10^{18}$ \\
$N_{V}\left[\mathrm{~cm}^{-3}\right]$ & $1.8 \times 10^{19}$ & $1.8 \times 10^{19}$ & $1.8 \times 10^{19}$ & $1.8 \times 10^{19}$ \\
$\mu_{n}\left[\mathrm{~cm}^{2} /(\mathrm{V} \cdot \mathrm{s})\right]$ & 100 & 100 & 100 & Variable \\
$\mu_{p}\left[\mathrm{~cm}^{2} /(\mathrm{V} \cdot \mathrm{s})\right]$ & 25 & 25 & 25 & Variable \\
$N_{D}\left[\mathrm{~cm}^{-3}\right]$ & $1 \times 10^{20}$ & Variable & Variable & 0 \\
$N_{A}\left[\mathrm{~cm}^{-3}\right]$ & 0 & 0 & 0 & Variable \\
\hline
\end{tabular}

Simulation of the device has been performed by setting, for each layer, the optical and electrical parameters in Table 1 , as reported in literature [28-30].

We have analysed different band-gap values, with the corresponding absorption spectra taken from Paulson et al. [19]. The band gap of the absorber surges from $1.04 \mathrm{eV}$ (pure CIS) 
TABLE 2: Simulation material Gaussian defect for the CIGS solar layers.

\begin{tabular}{lcccc}
\hline Parameter & ZnO:Al & ZnO & CdS & CIGS \\
\hline Defect type & Donor & Donor & Acceptor & Donor \\
Energy level $[\mathrm{eV}]$ & 1.65 & 1.65 & 1.2 & 0.6 \\
Deviation $[\mathrm{eV}]$ & 0.1 & 0.1 & 0.1 & 0.1 \\
$\sigma_{n}\left[\mathrm{~cm}^{2}\right]$ & $1 \times 10^{-12}$ & $1 \times 10^{-12}$ & $1 \times 10^{-17}$ & $5 \times 10^{-13}$ \\
$\sigma_{p}\left[\mathrm{~cm}^{2}\right]$ & $1 \times 10^{-15}$ & $1 \times 10^{-15}$ & $1 \times 10^{-12}$ & $1 \times 10^{-15}$ \\
$N_{t}\left[\mathrm{~cm}^{-3}\right]$ & $1 \times 10^{17}$ & $1 \times 10^{17}$ & $1 \times 10^{18}$ & $1 \times 10^{14}$ \\
\hline
\end{tabular}

TABLE 3: Contact parameters applied in the simulations.

\begin{tabular}{lcc}
\hline Parameter & Back contact & Front contact \\
\hline$\Phi_{B}[\mathrm{eV}]$ & Variable & 0 \\
$S_{n}[\mathrm{~cm} / \mathrm{s}]$ & $2 \times 10^{7}$ & $1 \times 10^{7}$ \\
$S_{p}[\mathrm{~cm} / \mathrm{s}]$ & $2 \times 10^{7}$ & $1 \times 10^{7}$ \\
Reflectivity & 0.8 & 0.05 \\
\hline
\end{tabular}

TABLE 4: Explanation of the parameters used during the simulations.

\begin{tabular}{lc}
\hline Parameter & Explanation \\
\hline$D$ & Layer thickness \\
$\varepsilon_{R}$ & Permittivity constant \\
$\chi$ & Electron affinity \\
$N_{C} / N_{V}$ & Effective density of states in the conduction/valence \\
$\mu_{n} / \mu_{p}$ & band \\
$\sigma_{n} / \sigma_{p}$ & Mobility of electrons/holes \\
$N_{D} / N_{A}$ & Capture cross section of electrons/holes \\
$N_{t}$ & Doping concentration \\
$\Phi_{B}$ & Defect concentration \\
$S_{n} / S_{p}$ & Potential barrier height \\
\hline
\end{tabular}

to $1.69 \mathrm{eV}$ (pure CGS) according to the following relationship [31]:

$$
E_{g}(x)=1.02+0.67 x+0.24 x(x-1),
$$

where $x$ is the Ga fraction in the absorber layer.

Tables 2 and 3 summarize material Gaussian defect states and contact parameters, respectively, while Table 4 provides an explanation of the symbols used in the previous tables. Finally, front and back contacts are defined by means of the corresponding metal work function. Numerical simulations have been performed by using the standard AM1.5G solar spectrum, a reflection coefficient on the top of the cell equal to $5 \%$ and a cell temperature of $300 \mathrm{~K}$.

It is worth noting that a stable and not considerably rectifying back-contact is needed in a CIGS solar cell to obtain good performance and stability. For this reason, the development of a low barrier back-contact and, hence, a low contact resistance is an ongoing challenge in the fabrication of high performance CIGS based solar cells.
In general, metal-to-semiconductor contacts can act either as a rectifying (Schottky) or as an ohmic contact, according to the properties of the interface. In detail, in the case of a p-type semiconductor having a band-gap $E_{g}$, electron affinity $\chi$, and a metal with work function $\Phi_{m}$, an ohmic metal/semiconductor contact is obtained when

$$
\Phi_{m}>E_{g}+\chi
$$

On the other hand, a rectifying contact is formed when the following relationship occurs:

$$
\Phi_{m}<E_{g}+\chi
$$

At the Schottky-contact interface, majority carriers (holes) see a barrier $\Phi_{B}$, as they travel from the semiconductor towards the metal, but such a barrier is not present in the case of an ohmic contact interface.

Most metals, however, do not have sufficiently high work functions and therefore form Schottky-barrier contacts: this is indeed the case for the p-CIGS absorber layers/Molybdenum interface.

In the case of an ideal contact between a metal and a ptype semiconductor, and in the absence of surface states, it is possible to express the contact barrier height for holes as follows [32]:

$$
\Phi_{B}=E_{g}+\chi-\Phi_{m}
$$

For the simulations that we have carried out, at varying $E_{g}$ we have assumed $E_{g}+\chi$ constant and equal to $5.55 \mathrm{eV}$ [33], and also $\Phi_{m}(\mathrm{Mo})=4.95 \mathrm{eV}$ [34], obtaining $\Phi_{B}=0.6 \mathrm{eV}$. In other words, our simulations were performed keeping the back-contact barrier $\Phi_{B}$ constant.

\section{Results and Discussion}

CIGS photovoltaic cells are typically composed of a $\mathrm{Cu}(\mathrm{In}, \mathrm{Ga}) \mathrm{Se}_{2}$ layer having a thickness of about $2 \div 3 \mu \mathrm{m}$. Decreasing such a thickness without reducing the performance too much would allow to cutting the deposition time of the CIGS thin-film and thus a considerable saving of the raw materials employed, with a substantial decrease of production costs. Moreover, decreasing the usage of Indium and Gallium during the mass production of CIGS solar cells would surely have positive ecological and environmental impacts. Nonetheless, there are some drawbacks caused by the reduction of the absorber layer thickness, reported in detail in [35-37].

By means of the aforementioned software, we have first performed some simulative campaigns of conventional CIGS cells (herein called Uniform Carrier Concentration Profile, UCCP). The simulations have been carried out at several different energy gap values, keeping constant the properties of the different layers while changing the absorber thickness, as shown in Figure 2. In this manner, it has been possible to investigate on the effect of the absorber layer thickness on the electrical parameters of the solar cell.

As reported in Figure 2, with a thickness greater than $2 \mu \mathrm{m}$, the solar cell absorbs most of the useful solar spectrum 


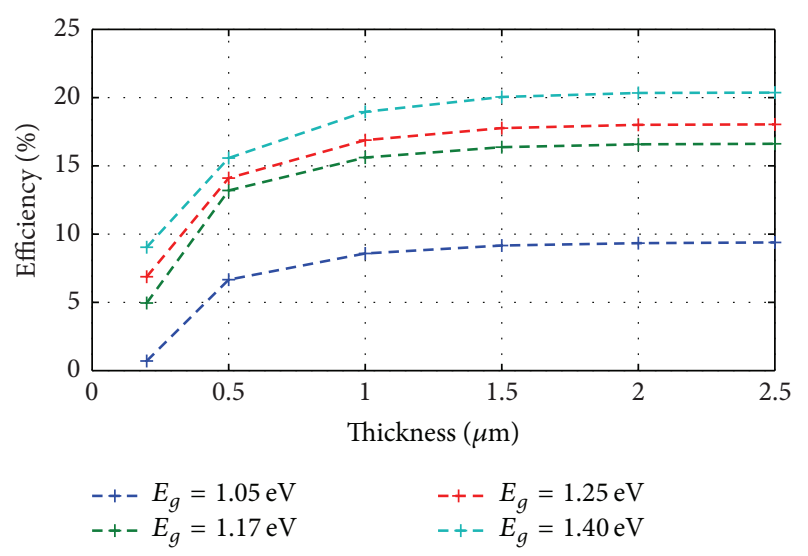

FIGURE 2: Efficiency versus CIGS absorber thickness at varying absorber energy gap values.

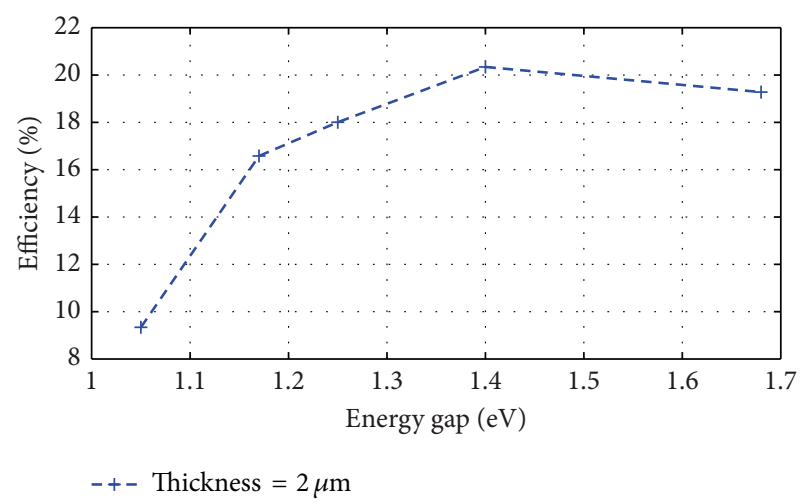

FIGURE 3: Efficiency of solar cell versus CIGS energy gap for a $2 \mu \mathrm{m}$ thick absorber layer.

which it is sensitive to and, thus, the efficiency is practically constant. In particular, we were able to obtain a value higher than $95 \%$ of the maximum efficiency possible when the absorber thickness is greater than $1 \mu \mathrm{m}$, regardless of the energy gap, that is, irrespective of the fraction of $\mathrm{Ga}$ in the CIGS.

In Figure 3 the efficiency versus energy gap curve is shown, going from a pure CIS composition $\left(E_{g}=1.02 \mathrm{eV}\right)$ to a CGS $\left(E_{g}=1.68 \mathrm{eV}\right)$, for a $3 \mu \mathrm{m}$ thick absorber layer. As reported, the absolute maximum efficiency is obtained for an energy gap value $\left(E_{g}\right)$ of about $1.4 \mathrm{eV}$, which corresponds to a $\mathrm{Ga} /(\mathrm{Ga}+\mathrm{In})$ ratio of 0.66 .

A similar behavior is observed for other thickness values, not reported here for brevity, and thus this trend happens regardless of the absorber thickness. Similarly, the maximum fill factor (FF), around $75 \%$, is obtained again for an $E_{g}=$ $1.4 \mathrm{eV}$.

Figure 4 depicts the variation of both $J_{\mathrm{SC}}$ and $V_{\mathrm{OC}}$ when increasing the energy gap from 1.02 to $1.68 \mathrm{eV}$. The obtained results show that, in the range under investigation, $J_{\mathrm{SC}}$ decreases almost linearly whereas the $V_{\text {OC }}$ increases.

By using this simple structure of conventional CIGS cells, it is possible to achieve a good efficiency only for high values

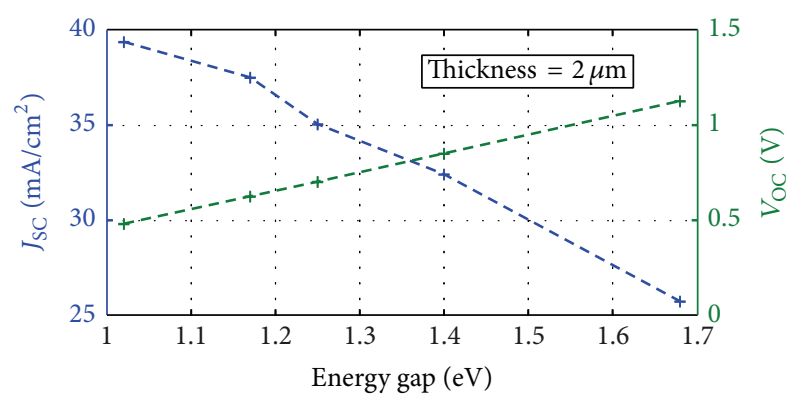

FIGURE 4: Short-circuit current density $J_{\text {SC }}$ and open-circuit voltage $V_{\mathrm{OC}}$ versus CIGS energy gap for a $2 \mu \mathrm{m}$ thick absorber layer.

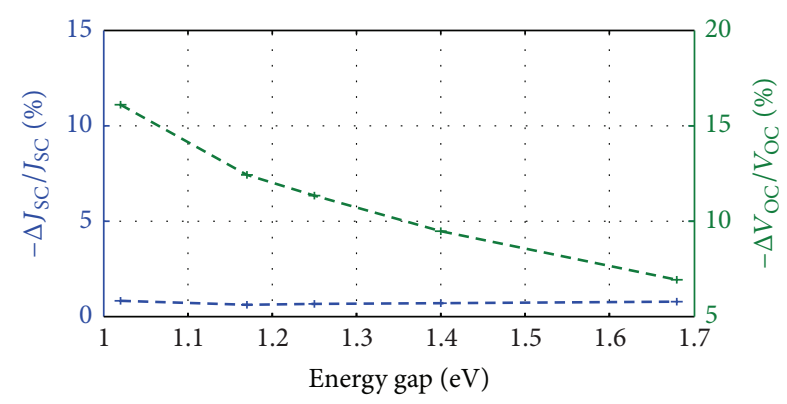

FIGURE 5: Relative variation of the short-circuit current density $J_{\mathrm{SC}}$ and the open-circuit voltage $V_{\mathrm{OC}}$ versus CIGS energy gap when employing the Mo back-contact.

of energy gap, as expected. However, augmenting the $E_{g}$ gap is not the ideal technological solution, since it causes an increased defect density in the areas presenting high $\mathrm{Ga}$ content [38]. Therefore, this high efficiency reported in the simulations cannot be achieved in a real device.

In addition, another reason that can reduce the performance in real devices consists in the presence of the back contact barrier at CIGS/Mo interface. In order to quantify this effect, we have performed some additional measurement campaigns comparing the device performances in the presence both of a real back-contact (i.e., Molybdenum) and an ideal back-contact. The ideal interface has been simulated with a surface recombination speed equal to the carrier thermal velocity and a barrier height equal to zero. The results are shown in Figures 5 and 6. In detail, Figure 5 depicts the relative variation of the short-circuit current density $J_{\mathrm{SC}}$ and the open-circuit voltage $V_{\mathrm{OC}}$ - at increasing energy gapwhen employing the real back-contact. The reference values ( $J_{\mathrm{SC}}$ and $\left.V_{\mathrm{OC}}\right)$ refers to the case with the Mo back-contact. Similarly, Figure 6 illustrates the variation of the efficiency and the fill factor. The results indicate that the open-circuit voltage decreases of about $7 \%$ at higher $E_{g}$ values, while the difference is even more pronounced for lower $E_{g}$ values (up to $16 \%)$. With regard to the short circuit current, there are no noticeable differences for the whole range of considered energy gaps. The fill factor decreases of just $2 \%$ at higher energy gaps, while the difference is more noticeable for lower $E_{g}$ absorbers (about 9\%). The same trend can be observed for the efficiency: for absorbers with lower energy gaps the Mo 


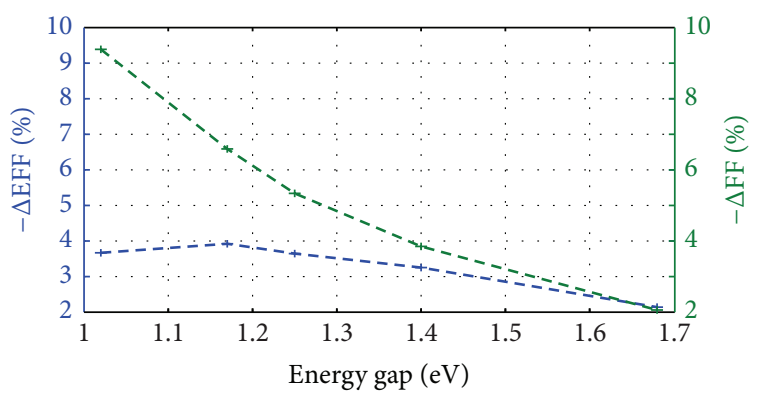

FIGURE 6: Variation of the efficiency and the fill factor versus CIGS energy gap when employing the Mo back-contact.

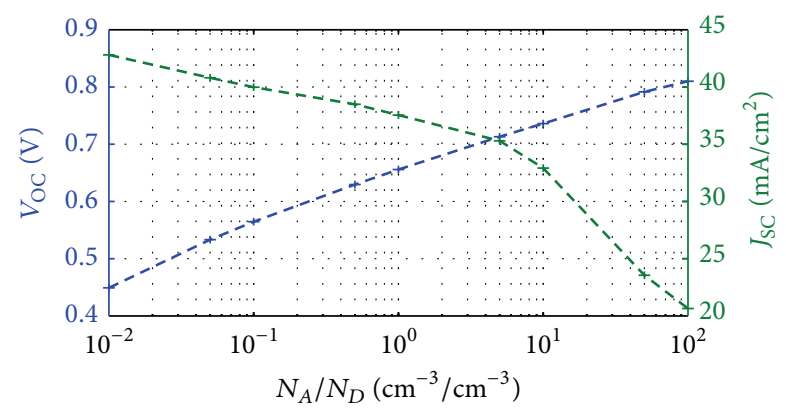

FIGURE 7: Open-circuit voltage and short circuit density versus the ratio between the $N_{A}$ concentration and the donors concentration $N_{D}$, for a given fixed value of $N_{D}=10^{17} \mathrm{~cm}^{-3}$.

real back-contact causes a decrease in efficiency of about $4 \%$, whereas this effect is less noticeable at increasing $E_{g}$.

From the above-mentioned results, it is clear that it would be convenient to use high energy gap absorbers, but this would cause an increase of density defects when augmenting the Ga content, as already before-mentioned. In order to overcome this limitation, in the literature other kinds of cells have been realized [39] having a variable absorber composition with an increased energy gap only in the area near the Mo back-contact. In particular, the above-mentioned cells possess, in the CIGS layer, a Ga fraction increasing from about $0.3\left(E_{g} \sim 1.2 \mathrm{eV}\right)$ near the junction up to $0.5\left(E_{g} \sim 1.4 \mathrm{eV}\right)$ near the Mo contact. In this way, it is possible to reach an efficiency slightly higher than $20 \%$.

In order to reduce the overall effect of the Molybdenum back-contact, acceptor doping concentration of absorber CIGS layer $N_{A}$ must be augmented. For this reason, in the literature [40], high efficiency cells have been recently obtained through an accurate control of the doping concentration of the absorber during its growth.

Figure 7 shows both the $V_{\mathrm{OC}}$ and the $J_{\mathrm{SC}}$ versus the ratio between the $N_{A}$ concentration and the donors concentration $N_{D}$, for a given fixed value of $N_{D}=10^{17} \mathrm{~cm}^{-3}$. As reported, $V_{\mathrm{OC}}$ increases with $N_{A}$, while $J_{\mathrm{SC}}$ decreases due to an increased recombination rate near the space charge region (SCR). Similar trends have been obtained for $N_{D}$ in the range between $10^{15}$ and $10^{18} \mathrm{~cm}^{-3}$ and are not reported here for brevity.

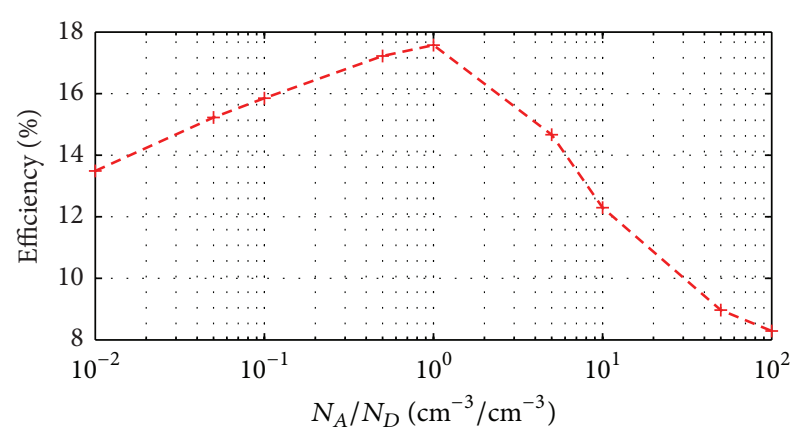

FIGURE 8: Efficiency versus the ratio between the $N_{A}$ concentration and the donors concentration $N_{D}$ for a given fixed value of $N_{D}=$ $10^{17} \mathrm{~cm}^{-3}$

As a consequence, the efficiency has a maximum value, as reported in Figure 8 that depicts the efficiency versus the ratio between the $N_{A}$ concentration and the donors concentration $N_{D}$.

In detail, the maximum is obtained when $N_{D}$ is almost equal to $N_{A}$. Also in this case, similar trends have been obtained for other values of $N_{D}$ in the range between $10^{15}$ and $10^{18} \mathrm{~cm}^{-3}$.

In order to improve both $V_{\mathrm{OC}}$ and $J_{\mathrm{SC}}$, we propose the innovative structure having a graded carrier concentration absorber profile, already described in Section 2.2 and herein called Graded Carrier Concentration Profile, GCCP.

In other words, we have simulated a CIGS solar cell with the absorber consisting of 10 different $0.2 \mu \mathrm{m}$-thick layers each one having a different doping concentration $\left(N_{A}\right)$. The overall CIGS absorber thickness (i.e., $2 \mu \mathrm{m}$ ) has been chosen to make it an easy comparison possible with the aboveanalysed conventional cell.

In detail, the doping concentration in the absorber is supposed to linearly increase towards the back contact, with the maximum value of $N_{A}$ in the layer located near the absorberMolibdenum interface. In our simulations, the minimum value of $N_{A}$ is supposed to be in the layer near the junction $\left(N_{A \_\mathrm{LJ}}\right)$ and varies from $10^{14} \mathrm{~cm}^{-3}$ to $10^{19} \mathrm{~cm}^{-3}$, while the maximum value of $N_{A}$ is in the layer placed near the absorber-Molibdenum interface $\left(N_{A_{\perp} M_{0}}\right)$ and it is assumed constant to $N_{\text {A_Mo }}=10^{19} \mathrm{~cm}^{-3}$. This upper bound has been chosen since it is very difficult, from a technological point of view, to realize higher $N_{A}$ concentrations. In addition, we want to underline that we will focus on an absorber having a $\mathrm{Ga}$ fraction equal to 0.31 , which can be easily realized with a better quality, contrarily to absorbers with higher Ga content.

In order to reveal more information on the working principle of such structures, we have first carried out a depthdependent investigation of our CIGS cells. In particular, in Figure 9, we depict the energy-band diagram versus depth for a GCCP cell, with $N_{D}=N_{A_{-} \mathrm{LJ}}=10^{17} \mathrm{~cm}^{-3}$, compared to a conventional uniform CIGS absorber profile, with $N_{D}=$ $N_{A}=10^{17} \mathrm{~cm}^{-3}$. As reported, the GCCP structure causes the energy band of the absorber to linearly increase towards the back contact. 


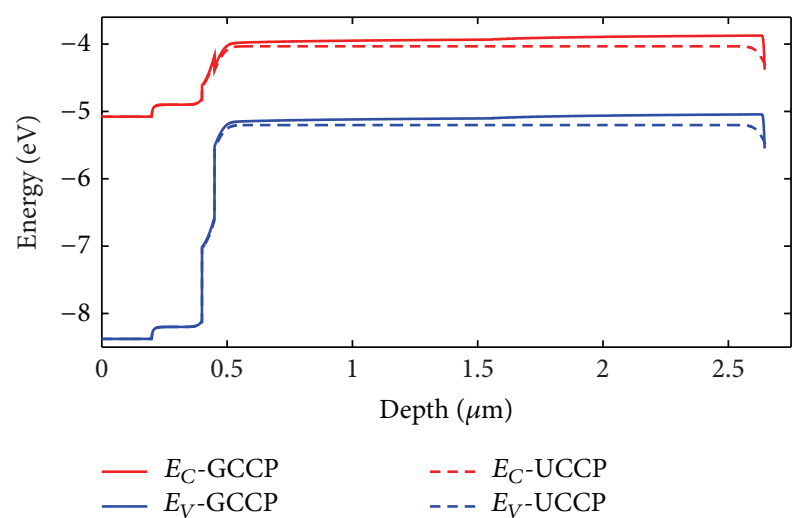

FIGURE 9: The schematic energy-band diagram versus depth of a $\operatorname{GCCP}\left(N_{D}=N_{A \perp J}=10^{17} \mathrm{~cm}^{-3}\right)$ and UCCP $\mathrm{ZnO}: \mathrm{Al} / \mathrm{ZnO} /$ CdS/CIGS/Mo solar cell under standard AM1.5G solar spectrum condition.

Therefore, similarly to what happens inside a CIGS cell having a Ga back graded profile, a quasielectrical field directed towards the back-contact is induced through the absorber. For the case shown in Figure 9 the value of the generated quasielectrical field is about $600 \mathrm{~V} / \mathrm{cm}$. This field causes the drift of the photogenerated electrons and of the holes, the first towards the SCR and the latter towards the back-contact, respectively. Therefore, the minority-carrier "drift-diffusion length" is increased, and thus an efficient collection of the carrier at the junction can be achieved in GCCP CIGS cells. This effect reduces both bulk and back surface recombination at the contact, and thus $J_{\mathrm{SC}}, V_{\mathrm{OC}}, \mathrm{FF}$, and the efficiency are enhanced.

However, an important difference compared to Ga back graded profile consists in the fact that in our structure both $E_{C}$ and $E_{V}$ increase towards the back-contact, and thus the energy gap keeps constant along the absorber depth. Thanks to this behaviour, the generation process is more efficient in the proposed cells if compared to Ga back-graded structures that present higher thermalization losses.

As depicted in Figure 10, due to the above-mentioned quasielectrical field, the minority carrier current direction changes just before the back-contact in a GCCP cell, when the back surface field at the CIGS/Mo interface becomes dominant. Instead, in an UCCP cell this change happens near the junction, and thus all the carriers photogenerated deeper inside the absorber contribute to reduce the cell performances. Hence, in our proposed GCCP solar cell the total current density increases compared to a conventional cell.

Using the above-mentioned GCCP structure, we have performed several simulation campaigns aimed at analysing how the main parameters (i.e., efficiency, short circuit current, and open circuit voltage) are improved if compared to the conventional structure.

The results are depicted in Figure 11, in which we show the efficiency versus the $N_{A_{\_} \mathrm{LJ}} / N_{D}$ ratio. In detail, $N_{A_{-} \mathrm{LJ}}$ is the concentration near the junction, while $N_{D}$ is the donors concentration of the two n-type junction layers (i.e., CdS and

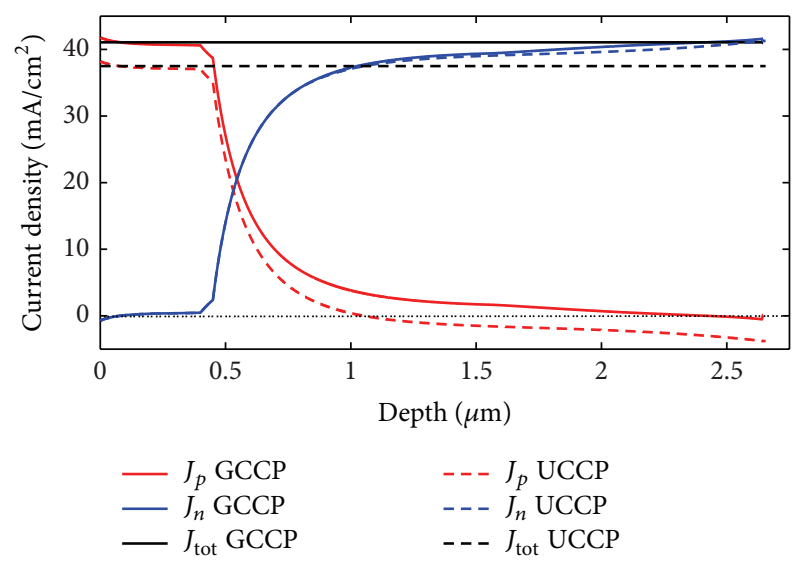

Figure 10: Current density versus depth of a GCCP $\left(N_{D}=N_{A \perp J}=\right.$ $10^{17} \mathrm{~cm}^{-3}$ ) and UCCP $\mathrm{ZnO} / \mathrm{CdS} / \mathrm{CIGS}$ solar cell under solar cell under standard AM1.5G solar spectrum condition.

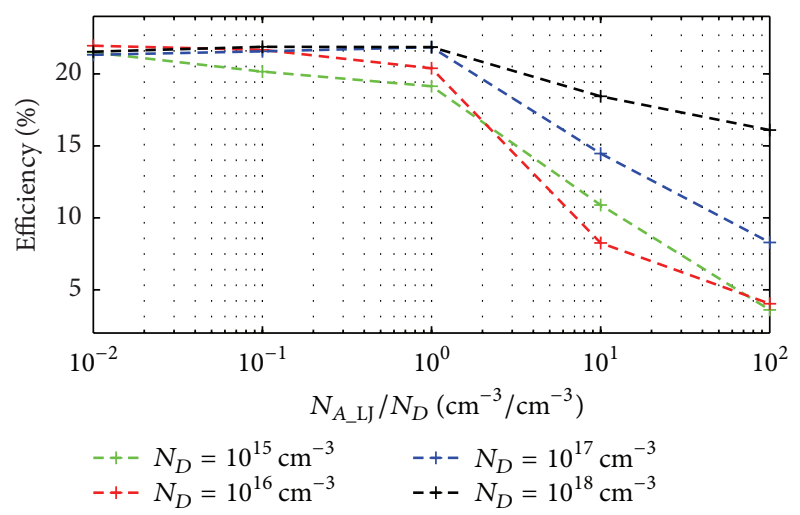

FIGURE 11: Efficiency versus the ratio between the $N_{A}$ concentration near the junction and the donors concentration $N_{D}$ at several different values of $N_{D}$.

$\mathrm{ZnO}$ ) composing the structure. Several different $N_{D}$ values were taken into account, ranging from $10^{15} \mathrm{~cm}^{-3}$ to $10^{18} \mathrm{~cm}^{-3}$.

As reported, by using the proposed cell structure and properly choosing the value of $N_{A}$ near the junction, it is possible to achieve high efficiency (up to about 22\%), regardless of the $N_{D}$ concentration. Comparing these values with the conventional CIGS structure, see Figure 3 at the corresponding $E_{g}=1.2 \mathrm{eV}$, a strong surge in efficiency (more than $4 \%$ ) can be observed. It is worth noting that this is true only if we have an $N_{A}$ near the junction less or equal to the $N_{D}$ concentration (i.e., $N_{A \perp \mathrm{LJ}} \leq N_{D}$ ); otherwise, the efficiency noticeably decreases due to the already mentioned photo carrier recombination effect close to the junction, which decreases the $J_{\mathrm{SC}}$, as reported in Figure 12. A similar trend is observed for the fill factor, not reported here for brevity.

Lastly, Figure 13 shows the $V_{\mathrm{OC}}$ versus $N_{A_{-} \mathrm{LJ}} / N_{D}$ ratio, for $N_{D}$ in the range between $10^{15}$ and $10^{18} \mathrm{~cm}^{-3}$. As reported, the $V_{\mathrm{OC}}$ increases with the doping concentration. 




FIGURE 12: $J_{\text {SC }}$ versus the ratio between the $N_{A}$ concentration near the junction and the donors concentration $N_{D}$ at several different values of $N_{D}$.

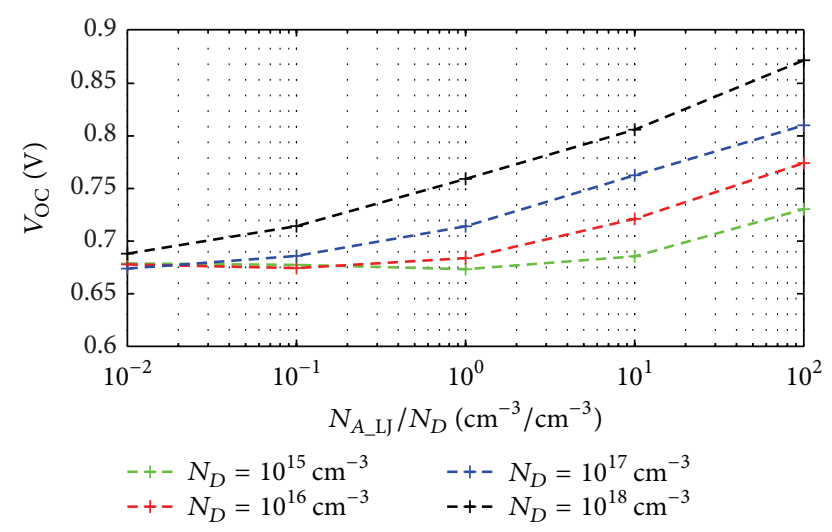

FIGURE 13: $V_{\mathrm{OC}}$ versus the ratio between the $N_{A}$ concentration near the junction and the donors concentration $N_{D}$ at several different values of $N_{D}$.

\section{Conclusions}

In this study, we have performed some simulative campaigns by means of the wxAMPS software, to investigate on the effects of the absorber band gap on the main electrical parameters in conventional CIGS solar cells. In detail, our results show that the efficiency increases with energy gap, up to a maximum peak around $1.40 \mathrm{eV}$. In addition, the Ga content also influences $V_{\mathrm{OC}}$ e $J_{\mathrm{SC}}$ : in detail, $V_{\mathrm{OC}}$ increases linearly with $E_{g}$, while $J_{\mathrm{SC}}$ decreases considerably. Furthermore, we have shown that all the electrical parameters remain almost constant when the absorber thickness is greater than $2 \mu \mathrm{m}$. Moreover, we have investigated on the effects of the molybdenum back-contact on the above-mentioned parameters, showing a decrease in efficiency of about $3-5 \%$ in presence of the back-contact, especially at lower values of Ga content.

However, the obtained efficiency is quite lower if compared to other kinds of cell already reported in the literature. For this reason, we have proposed a novel structure with a graded doping concentration absorber profile. A depthdependent investigation has shown that, in GCCP structures, a quasielectrical field directed towards the back-contact is induced through the absorber, $E_{C}$ and $E_{V}$ increase towards the back-contact, and thus the energy gap keeps constant along the depth. Therefore, the generation process is more efficient in GCCP cells compared to Ga back-graded structures. Close to the Mo back contact, the $N_{A}$ concentration should be the highest technologically feasible, while the $N_{A}$ concentration near the junction should be lower than $N_{D}$ to avoid an excessive photo carrier recombination effect. In this way, we were able to increase the efficiency up to over $21 \%$.

These results are of great importance to allow technological optimization during future experimental work.

\section{Conflict of Interests}

The authors declare that there is no conflict of interests regarding the publication of this paper.

\section{Acknowledgments}

This activity was supported by PON 011725 "Nuove Tecnologie Fotovoltaiche per Sistemi Intelligenti Integrati in Edifici" and PON 0200355 “Tecnologie per l'ENERGia e l'Efficienza energETICa-ENERGETIC" Research Programs. Contributions came also from SDESLab, University of Palermo.

\section{References}

[1] T. Unold and C. A. Kaufmann, "1.18. Chalcopyrite thin-film materials and solar cells," in Comprehensive Renewable Energy, E.-C. A. Sayigh, Ed., pp. 399-422, Elsevier, Oxford, UK, 2012.

[2] V. S. Saji, I.-H. Choi, and C.-W. Lee, "Progress in electrodeposited absorber layer for $\mathrm{CuIn}_{(1-x)} \mathrm{Ga}_{x} \mathrm{Se}_{2}$ (CIGS) solar cells," Solar Energy, vol. 85, no. 11, pp. 2666-2678, 2011.

[3] W. Witte, M. Powalla, D. Hariskos et al., "Chemical gradients in $\mathrm{Cu}(\mathrm{In}, \mathrm{Ga})(\mathrm{S}, \mathrm{Se})_{2}$ thin-film solar cells: results of the GRACIS project," in Proceedings of the 27th European Photovoltaic Solar Energy Conference and Exhibition, pp. 2166-2173, Frankfurt, Germany, 2012.

[4] "Achievements and challenges of solar electricity from photovoltaics," in Handbook of Photovoltaic Science and Engineering, A. Luque and S. Hegedus, Eds., John Wiley \& Sons, 2nd edition, 2011.

[5] A. Tomasino, A. Parisi, S. Stivala et al., "Wideband THz time domain spectroscopy based on optical rectification and electrooptic sampling," Scientific Reports, vol. 3, article 3116, pp. 1-8, 2013.

[6] A. Pasquazi, A. Busacca, S. Stivala, R. Morandotti, and G. Assanto, "Nonlinear disorder mapping through three-wave mixing," IEEE Photonics Journal, vol. 2, no. 1, pp. 18-28, 2010.

[7] A. Parisi, A. C. Cino, A. C. Busacca, M. Cherchi, and S. RivaSanseverino, "Integrated optic surface plasmon resonance measurements in a borosilicate glass substrate," Sensors, vol. 8, no. 11, pp. 7113-7124, 2008.

[8] R. Pernice, G. Adamo, S. Stivala et al., "Opals infiltrated with a stimuli-responsive hydrogel for ethanol vapor sensing," Optical Materials Express, vol. 3, no. 11, pp. 1820-1833, 2013.

[9] M. Cherchi, S. Bivona, A. C. Cino, A. C. Busacca, and R. L. Oliveri, "Universal charts for optical difference frequency generation in the terahertz domain," IEEE Journal of Quantum Electronics, vol. 46, no. 6, pp. 1009-1013, 2010. 
[10] Y. Goushi, H. Hakuma, K. Tabuchi, S. Kijima, and K. Kushiya, "Fabrication of pentanary $\mathrm{Cu}(\mathrm{InGa})(\mathrm{SeS}) 2$ absorbers by selenization and sulfurization," Solar Energy Materials and Solar Cells, vol. 93, no. 8, pp. 1318-1320, 2009.

[11] B. B. Vermang, J. T. Wätjen, V. Fjällström et al., "Highly reflective rear surface passivation design for ultra-thin $\mathrm{Cu}(\mathrm{In}, \mathrm{Ga}) \mathrm{Se}_{2}$ solar cells," Thin Solid Films, vol. 582, pp. 300-303, 2015.

[12] M. Gloeckler and J. R. Sites, "Band-gap grading in $\mathrm{Cu}(\mathrm{In}, \mathrm{Ga}) \mathrm{Se}_{2}$ solar cells," Journal of Physics and Chemistry of Solids, vol. 66, no. 11, pp. 1891-1894, 2005.

[13] N. E. Gorji, M. D. Perez, U. Reggiani, and L. Sandrolini, "A new approach to valence and conduction band grading in CIGS thin film solar cells," International Journal of Engineering and Technology, vol. 4, no. 5, pp. 573-576, 2012.

[14] J. Mattheis, P. J. Rostan, U. Rau, and J. H. Werner, "Carrier collection in $\mathrm{Cu}(\mathrm{In}, \mathrm{Ga}) \mathrm{Se}_{2}$ solar cells with graded band gaps and transparent ZnO:Al back contacts," Solar Energy Materials and Solar Cells, vol. 91, no. 8, pp. 689-695, 2007.

[15] R. Inguanta, S. Piazza, C. Sunseri et al., "An electrochemical route towards the fabrication of nanostructured semiconductor solar cells," in Proceedings of the International Symposium on Power Electronics, Electrical Drives, Automation and Motion (SPEEDAM '10), pp. 1166-1171, IEEE, June 2010.

[16] A. Parisi, L. Curcio, V. Rocca et al., "Thin film CIGS solar cells, photovoltaic modules, and the problems of modeling," International Journal of Photoenergy, vol. 2013, Article ID 817424, 12 pages, 2013.

[17] A. C. Busacca, V. Rocca, L. Curcio et al., "Parametrical study of multilayer structures for CIGS solar cells," in Proceedings of the International Conference on Renewable Energy Research and Application (ICRERA '14), pp. 964-968, Milwaukee, Wis, USA, October 2014.

[18] G. Cipriani, V. Di Dio, R. Miceli et al., "CIGS PV module characteristic curves under chemical composition and thickness variations," in Proceedings of the International Conference on Renewable Energy Research and Applications (ICRERA '14), pp. 969-973, Milwaukee, Wis, USA, October 2014.

[19] P. D. Paulson, R. W. Birkmire, and W. N. Shafarman, "Optical characterization of $\mathrm{CuIn}_{1-x} \mathrm{Ga}_{x} \mathrm{Se}_{2}$ alloy thin films by spectroscopic ellipsometry," Journal of Applied Physics, vol. 94, no. 2, pp. 879-888, 2003.

[20] N. Hernández-Como and A. Morales-Acevedo, "Simulation of hetero-junction silicon solar cells with AMPS-1D," Solar Energy Materials and Solar Cells, vol. 94, no. 1, pp. 62-67, 2010.

[21] H. Zhu, A. K. Kalkan, J. Hou, and S. J. Fonash, "Applications of AMPS-1D for solar cell simulation," AIP Conference Proceedings, vol. 462, pp. 309-314, 1999.

[22] P. A. Basore and D. A. Clugston, "PC1D version 4 for windows: from analysis to design," in Proceedings of the 25th IEEE Photovoltaic Specialists Conference, pp. 377-381, May 1996.

[23] S. Degrave, M. Burgelman, and P. Nollet, "Modelling of polycrystalline thin film solar cells: new features in scaps version 2.3," in Proceddings of the 3rd World Conference on Photovoltaic Energy Conversion, pp. 487-490, IEEE, Osaka, Japan, May 2003.

[24] S. Fonash, J. Arch, J. Cuifi et al., A Manual for AMPS-1D for Windows 95/NT, Pennsylvania State University, State College, $\mathrm{Pa}$, USA, 1997.

[25] Y. Liu, Y. Sun, and A. Rockett, "An improved algorithm for solving equations for intra-band tunneling current in heterojunction solar cells," Thin Solid Films, vol. 520, no. 15, pp. 49474950, 2012.
[26] https://wiki.engr.illinois.edu/display/solarcellsim.

[27] B. J. Stanbery, "Copper indium selenides and related materials for photovoltaic devices," Critical Reviews in Solid State and Materials Sciences, vol. 27, no. 2, pp. 73-117, 2002.

[28] Z. Jehl, F. Erfurth, N. Naghavi et al., "Thinning of CIGS solar cells: part II: cell characterizations," Thin Solid Films, vol. 519, no. 21, pp. 7212-7215, 2011.

[29] A. Niemegeers, S. Gillis, and M. Burgelman, "A user program for realistic simulation of polycristalline heterojunction solar cells: SCAPS-1D," in Proceedings of the 2nd World Conference on Photovoltaic Energy Conversion, Wien, Austria, July 1998.

[30] R. Scheer, "Towards an electronic model for $\mathrm{CuIn}_{1-x} \mathrm{Ga}_{x} \mathrm{Se}_{2}$ solar cells," Thin Solid Films, vol. 519, no. 21, pp. 7472-7475, 2011.

[31] O. Lundberg, M. Edoff, and L. Stolt, "The effect of Ga-grading in CIGS thin film solar cells," Thin Solid Films, vol. 480-481, pp. 520-525, 2005.

[32] S. M. Sze, Physics of Semiconductor Devices, Wiley, 2nd edition, 1981.

[33] Y. Hirai, Y. Kurokawa, and A. Yamada, "Numerical study of $\mathrm{Cu}(\mathrm{In}, \mathrm{Ga}) \mathrm{Se}_{2}$ solar cell performance toward $23 \%$ conversion efficiency," Japanese Journal of Applied Physics, vol. 53, no. 1, Article ID 012301, 2014.

[34] P. Ranade, H. Takeuchi, T.-J. King, and C. Hu, "Work function engineering of molybdenum gate electrodes by nitrogen implantation," Electrochemical and Solid-State Letters, vol. 4, no. 11, pp. G85-G87, 2001.

[35] M. Gloeckler, A. L. Fahrenbruch, and J. R. Sites, "Numerical modeling of CIGS and CdTe solar cells: setting the baseline," in Proceedings of the 3rd World Conference on Photovoltaic Energy Conversion, vol. 1, pp. 491-494, IEEE, Osaka, Japan, May 2003.

[36] P. Jackson, D. Hariskos, E. Lotter et al., "New world record efficiency for $\mathrm{Cu}(\mathrm{In}, \mathrm{Ga}) \mathrm{Se}_{2}$ thin-film solar cells beyond $20 \%$," Progress in Photovoltaics: Research and Applications, vol. 19, no. 7, pp. 894-897, 2011.

[37] M. Gloeckler and J. R. Sites, "Potential of submicrometer thickness $\mathrm{Cu}(\mathrm{In}, \mathrm{Ga}) \mathrm{Se}_{2}$ solar cells," Journal of Applied Physics, vol. 98, Article ID 103703, 2005.

[38] G. Hanna, A. Jasenek, U. Rau, and H. W. Schock, "Influence of the Ga-content on the bulk defect densities of $\mathrm{Cu}(\mathrm{In}, \mathrm{Ga}) \mathrm{Se}_{2}$," Thin Solid Films, vol. 387, no. 1-2, pp. 71-73, 2001.

[39] C. L. Perkins, B. Egaas, I. Repins, and B. To, "Quantitative analysis of graded $\mathrm{Cu}\left(\mathrm{In}_{1-x}, \mathrm{Ga}_{x}\right) \mathrm{Se}_{2}$ thin films by AES, ICPOES, and EPMA," Applied Surface Science, vol. 257, no. 3, pp. 878-886, 2010.

[40] P. Salomè, V. Fjällström, A. Hultqvist, and M. Edoff, "Na doping of CIGS solar cells using low sodium-doped mo layer," IEEE Journal of Photovoltaics, vol. 3, no. 1, pp. 509-513, 2013. 

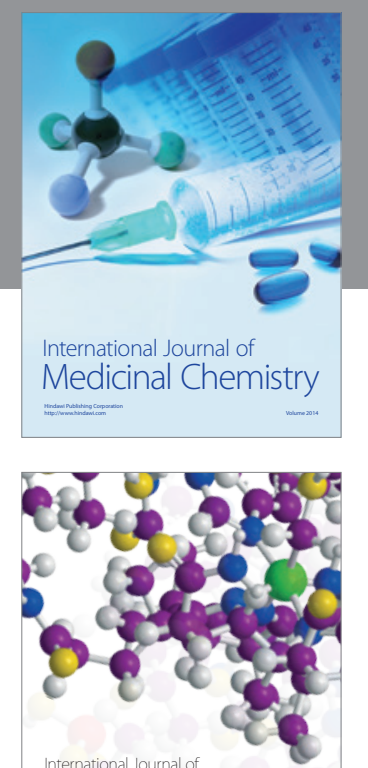

\section{Carbohydrate} Chemistry

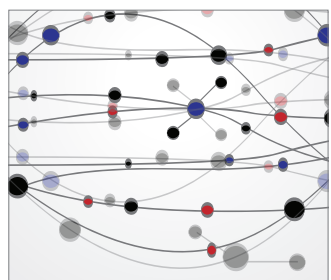

The Scientific World Journal
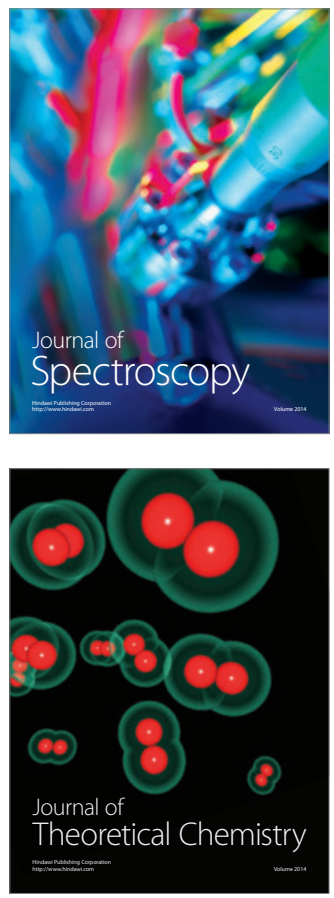
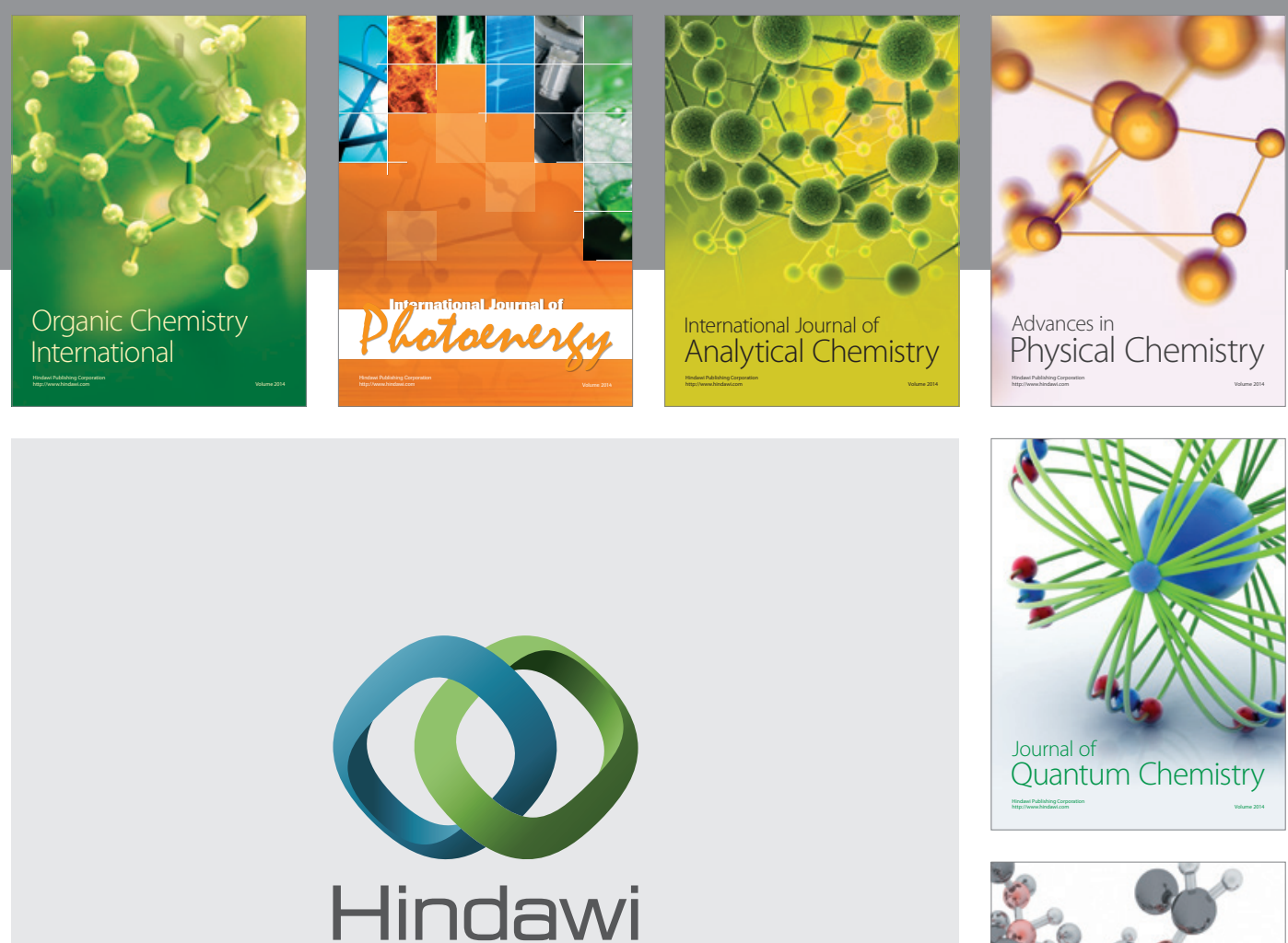

Submit your manuscripts at

http://www.hindawi.com

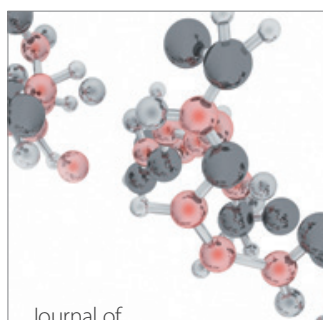

Analytical Methods

in Chemistry

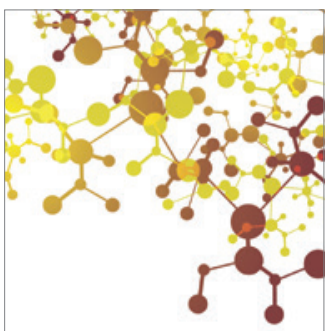

Journal of

Applied Chemistry

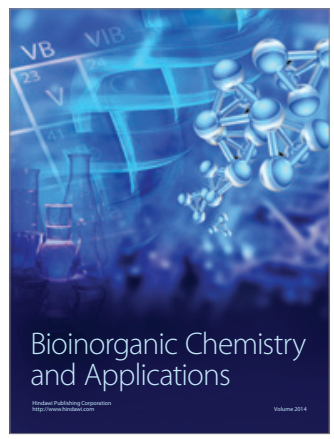

Inorganic Chemistry
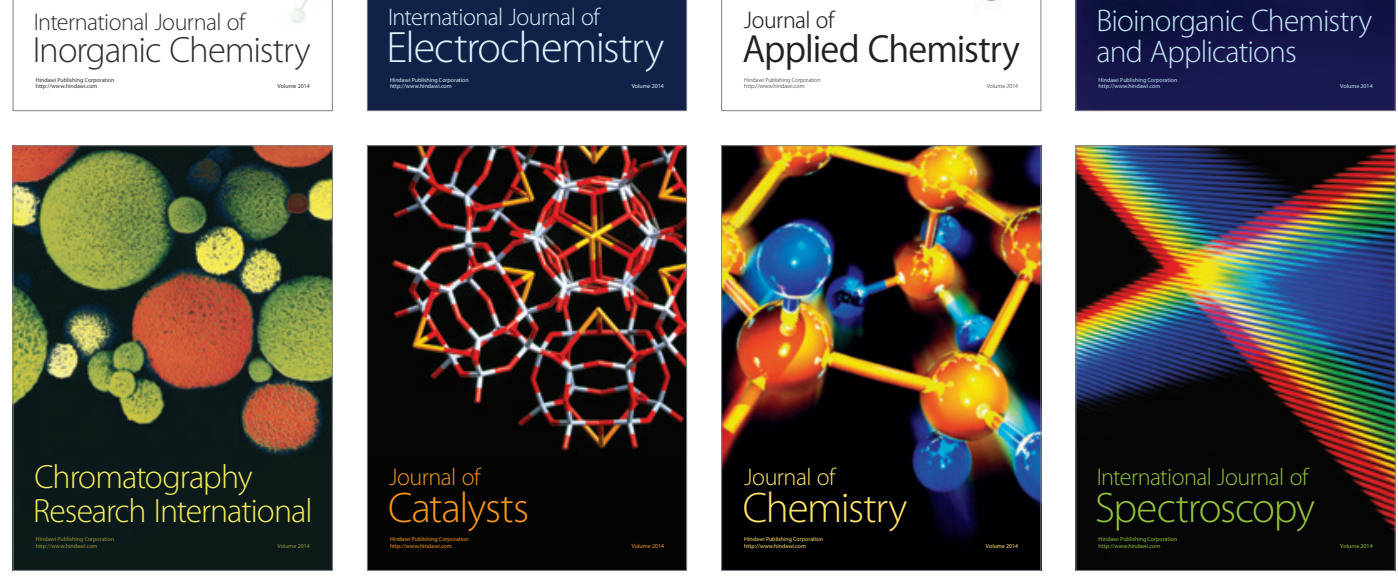